\title{
Private Governance in der Spielzeugindustrie* \\ Voraussetzungen und Strategien zur Durchsetzung einer
Branchenvereinbarung
}

\author{
REINHARD BIEDERMANN ${ }^{* *}$
}

\section{Private Governance and Social Standards in the Toy Industries}

In 2004, the global toy industry around its leading producers Mattel and Hasbro fortified its commitment to enforce common social standards in the worldwide toy factories with focus on China, where more than $70 \%$ of the global production takes place. Since then, the code of conduct of the International Council of the Toy Industries (ICTI) developed to a widely accepted common set of standards in the global supply chain. It aims at ending the implementation difficulties with too many different standards, but also gives small- and mediumsized companies the possibility to behave in a more social responsible way. The success of the common industrial standards might be a model for other globalized industry sectors that face similar challenges with labour protection, when the involvement of the workers is strenghtened in the future.

Keywords: Corporate Social Responsibility, Cooperation Theory, Private Governance, Social Standards, Toy Industry

\section{Branchenstandards durch Private Governance: Das Beispiel Sozialstan- dards in der Spielzeugbranche}

Private Governance durch multinationale Unternehmen und über nationale Grenzen hinweg hat seit den 1990er Jahren stark zugenommen (vgl. Cutler, Haufler, Porter 1999; Haufler 2001; Fuchs 2004; Hall, Biersteker 2002; Nölke 2004; Wilkinson 2005). In der Politikwissenschaft wird Private Governance in ihren verschiedenen Formen, ausgeübt durch Non-Profit-Organisationen, Verbände und multinationale Unternehmen untersucht, deren gemeinsames charakteristisches Kriterium Entscheidungen betrifft, mit der Teilnehmer nicht nur sich selbst, sondern auch andere Akteure binden (vgl. Rudder 2008: 899). Insbesondere durch grenzüberschreitende Sozialstandards im Welthandel wurde eine Vielzahl von privaten Initiativen von einzelnen Unternehmen, Multistakeholderinitiativen, von Public Private Partnerships (einer Spielart von Private Governance) oder von ganzen Branchen auf den Weg gebracht. Der öffentlichkeits-

\footnotetext{
Beitrag eingereicht am 31.03.2008; nach doppelt verdecktem Gutachterverfahren überarbeitete Fassung angenommen am 02.02.2009. Der Autor bedankt sich bei zwei anonymen Gutachtern für ihre hilfreichen Hinweise.

** Dr. Reinhard Biedermann, Institutum Iurisprudentiae/Academia Sinica, Nankang Road, Taipei, Taiwan, Tel.: +886-(0)2652-5426, E-Mail: reinhard@biedermann.de, Forschungsschwerpunkte: Sozialstandards im Welthandel, Private Governance, Kooperationstheorie, Politik Chinas, Europäische China-Beziehungen.
} 
wirksame Vorwurf von Nichtregierungsorganisationen und Medien, durch SweatshopArbeit zu profitieren, hat bei zahlreichen globalisierten Branchen und deren Marktführern zum Bekenntnis der Einhaltung von Mindeststandards und zum besseren Schutz der Arbeiter in Schwellen- und Entwicklungsländer geführt (vgl. Haufler 2001; Rowe 2004: 138). Bekannte Markenunternehmen und Verbände aus arbeitsintensiven, stark globalisierten Branchen, deren Vermarktung und Produktion ein starkes Nord-SüdGefälle ausweisen, haben sich Verhaltenskodizes gegeben, die dem Arbeitsschutz in Produktionsstätten dienen sollen. Obwohl es eine Vielzahl an Beispielen von Brachenvereinbarungen gibt, ${ }^{1}$ liegt es auch an der kontroversen Thematik, dass es keine Beispiele gibt, die einhellig sowohl von NGOs, Unternehmen, als auch Wissenschaft unterstützt werden. ${ }^{2}$

Als typisches Beispiel für diese komplexe Problematik hinsichtlich Durchsetzung und Bewertung untersucht dieser Artikel die Rolle des Branchenkodex in der Spielzeugindustrie zur globalen Durchsetzung von sozialen Mindeststandards. In der Spielzeugindustrie betrifft soziale Unternehmensverantwortung durch Private Governance in erster Linie den Konsumentenschutz, d. h. die Branche muss sicherstellen, dass Spielzeug sicher und nicht gesundheitsgefährdend für Kinder ist. Bis zu den großen Rückrufaktionen führender Hersteller und Händler von Millionen von Spielzeugartikeln in 2007 und 2008 ging der Weltverband der Spielzeugindustrie ICTI (International Council of the Toy Industries) davon aus, dass Sicherheits- und Gesundheitsstandards bei Spielzeugartikeln in der hochregulierten Branche in der Produktion beachtet werden, was sich jedoch als Trugschluss herausstellte. Zweites Kernelement sozialer Unternehmensverantwortung in der Branche sind die Arbeitsbedingungen in China, wo zwischen 70 und 80 Prozent des weltweit verkauften Spielzeugs hergestellt wird. Bereits 1995 gab sich der Weltspielzeugverband ICTI einen Branchenstandard, der grundlegende Mindeststandards zu Arbeiterrechten zu regeln beabsichtigt. Doch erst seit 2003 bzw. 2004 erfährt der Verhaltenskodex eine zunehmende Akzeptanz in der Branche. Dies ist insofern erstaunlich, da die „Hochphase“ der kritischen Auseinandersetzung der Zivilgesellschaft mit Sweatshops ihren Höhepunkt bereits überschritten $\mathrm{zu}$ haben scheint, was für eine gewisse Eigendynamik in der Branche spricht. Die

So hat sich 1987 die Initiative der globalen Chemieindustrie aus 45 Ländern nach der Chemiekatastrophe im indischen Bhopal zu einem branchenweiten Verhaltenskodex bekannt. 1993 gab sich der International Council on Mining and Metals eine branchenspezifische Charter zur Verringerung der häufigen Minenunfälle. 1995 hat sich die International Union of Food (IUF) einen Verhaltenskodex für den Teeanbau gegeben, 1997 hoben US-amerikanische Textilunternehmen und 19 kontinentalamerikanische Landesverbände die Worldwide Responsible Apparel Production Principles (WRAP) aus der Taufe. Der World Diamond Council gab sich 2000 einen Kodex als Antwort auf die „Blutdiamanten"-Vorwürfe. Der Weltverband der Sportartikelbranche gab sich ebenfalls 2000 einen Model Code of Conduct, und 2005 beschlossen die global führenden Elektronikkonzerne ihren Electronic Industry Code of Conduct (Biedermann 2007: 44), um nur einige zu nennen.

2 Aus politikwissenschaftlicher Sicht wird insbesondere kritisiert, dass sich Unternehmen parallel $\mathrm{zu}$ ihren privaten Initiativen gegen staatliche und internationale Regulierung positionieren, wodurch möglicherweise effizientere Regulierungen vermieden werden (vgl. Brühl et al. 2001; Scherrer; Greven 2001). Eine anders gelagerte Argumentation führt an, dass Unternehmen möglicherweise gerade dort Regulierungslücken füllen, wo Staaten ohnehin nicht mehr hinkommen (vgl. Risse 2005). 
Schwächen, die Branchenvereinbarungen auszeichnen, nämlich mangelnde Unterstützung durch die Branchenführer und eine unzureichende Institutionalisierung, werden seit einigen Jahren in der Spielzeugbranche angegangen: sämtliche Marktführer aus Industrie und zunehmend auch aus dem Handel, unterstützen den Branchenkodex (vgl. Biedermann 2006, 2007). Der Weltspielzeugverband hat ein Governance Board eingerichtet, das über Fortschritte und Schwächen berichtet und verantwortlich unabhängige, professionelle Monitoring-Agenturen akkreditiert, welche die ICTI-Standards in den Produzentenbetrieben kontrollieren. Die Branche hält sich zugute, dass heute mehr als eine Million Arbeiter in den Spielzeugfabriken Kantons in China von verbesserten Arbeitsbedingungen durch den ICTI-Kodex profitieren. Dieser Artikel untersucht, wie es zur Ausbreitung des Branchenstandards kam, einer Entwicklung, die beispielgebend auch für andere Branchen sein könnte.

\section{Pro und Contra Branchenvereinbarungen}

Grundsätzlich bestehen durch kooperative Branchenansätze durch Verhaltenskodizes zur Regelung der Arbeitsbeziehungen Vorteile gegenüber unilateralen Ansätzen, wenn die Verantwortung für die Durchsetzung von Sozialstandards alle Akteure gleichermaßen bindet, wodurch eine Art „Soft Law“ oder „Private Law“ (vgl. Cutler; Haufler; Porter 1999; Sethi 2003) entstehen kann. Zu weiteren Vorteilen gehören die branchenspezifische Regulierung sowie eine mögliche wettbewerbsneutrale Übernahme der Kosten durch alle Unternehmen einer Branche, die schließlich auf die Verbraucher abgewälzt werden können. Die klare Verantwortungszuweisung und schließlich die Chance einer branchenweiten Ausdehnung auch auf mittlere und kleinere Unternehmen im Norden und im Süden sind weitere Vorteile, die in der Debatte, wie diese in CSR-Schemen integriert werden können, eine praktikable Lösung sein können. Branchenvereinbarungen müssen die Interessen sehr vieler Akteure berücksichtigen. Auch jener Akteure im Süden, was die Übereinkunft von Branchenstandards erschwert. Um eine größtmögliche Interessenübereinstimmung zu erreichen, müssten Unternehmen, Regierungen, Verbände und Nichtregierungsorganisationen einen gemeinsamen Branchenansatz unterstützen, was im letzten Jahrzehnt bei globalen Standards jedoch auch zunehmend geschah (vgl. Krueger 2008: 114). Branchenstandards können auch für imagebewusste Marktführer interessant werden, wenn sie Zielscheibe von Kritik aus Medien und Zivilgesellschaft bleiben, obwohl sie bereits einen anspruchsvollen unilateralen Ansatz durch unternehmenseigene Verhaltenskodizes verfolgen, aber vorzugsweise von NGOs immer wieder öffentlichkeitswirksam an den Pranger gestellt werden (vgl. Krueger 2008: 116).

Branchenvereinbarungen haben allerdings auch den Ruf, „, nach unten verwässert“ zu sein, wenn der Langsamste die Qualität der Standards vorgibt (vgl. Sethi 2003), sodass letztlich nur so niedrige Standards gesetzt werden, deren Durchsetzung letztlich keine wirklichen Anstrengungen bedeuten (vgl. allgemein dazu Downs et al. 1995). Aus wettbewerbstheoretischer Sicht treten Unternehmen nicht nur mit ihren Produkten in die Konkurrenz, sondern eben auch mit ihrer Marke und ihrem Image, sodass sozial besonders aktive Unternehmen „First-Mover-Vorteile“ generieren könnten (vgl. Tiemann 1999). Für Marktführer kann es deshalb attraktiver sein, einen unilateralen Ansatz zu verfolgen, wodurch die wichtigsten Akteure einer Branchenvereinbarung fern- 
bleiben könnten. So raten Unternehmensberatungen im Bereich Corporate Social Responsibility kleineren und mittleren Unternehmen immer noch, eine eigene Strategie zu verfolgen, was angesichts der empirischen Wirklichkeit, vermehrt kooperativ handeln zu müssen, auf Unverständnis stoßen muss. ${ }^{3}$ Ein weiteres Problem bei Branchenvereinbarungen kann sich auch darin äußern, dass eben gerade die schwächsten Unternehmen, auf welche die Vorwürfe der NGOs am meisten zutreffen, branchenweiten Vereinbarungen fernbleiben (vgl. Fuchs 2004).

Problematisch bei Branchenvereinbarungen ist somit grundsätzlich die Frage, wie kollektives Handeln zu erreichen ist und welche individuellen Anreize bei einzelnen Akteuren bestehen, sich an kollektive Vereinbarungen zu halten. Die Frage, wie Kooperation unter Anarchie (cooperation under anarchy bei Axelrod/Keohane 1985) erreicht werden kann, ist in einem stark globalisierten Markt schwerer zu beantworten, als in einem nationalen oder im europäischen Umfeld. Denn in einem globalen, unregulierten Markt befindet sich eine nahezu unüberschaubare Anzahl an Akteuren, deren Bindung an koordinierende Verbände weit geringer ist, als im nationalen Verbandsund Regulierungsstaat. Große Handelskonzerne haben zum Teil viele tausend Lieferanten aus Entwicklungsländern, sowie viele Markenlieferanten, die wiederum in Schwellen- und Entwicklungsländern produzieren lassen. Produzenten, die Standards beachten, nehmen oft Rückgriff auf versteckte Sublieferanten mit niedrigeren Standards, um ihre Wettbewerbsfähigkeit zu erhalten. Ein weiteres zentrales Problem ist, wie sichergestellt wird, dass alle Akteure ihren eigenen Beitrag leisten und Trittbrettfahrer ausgeschaltet bzw. nicht belohnt werden. Denn nur die wenigsten Akteure in Produzentenländern werden dazu bereit sein, einen eigenen Beitrag zur Bereitstellung eines Kollektivgutes zu leisten, wenn sie befürchten müssen, dass direkte Konkurrenten dies nicht tun durchkommen und sogar durch Aufträge belohnt werden, weil sie billiger produzieren können. Machtvolle Akteure sowie mehr oder minder unabhängige Institutionen könnten glaubwürdige Zusagen machen und glaubhafte Abschreckungen organisieren, um Standards durchzusetzen (credible commitments und believable threats in der Kooperationsliteratur, vgl. Spar 1994). Ähnlich müssten bei Branchenvereinbarungen unabhängige Institutionen für eine Überwachung der Standards sorgen. Die Durchsetzung von Branchenstandards ist somit ein anspruchsvolles Unterfangen, dessen Voraussetzungen zunächst kooperationstheoretisch erörtert werden sollen.

\section{Branchenstandards durch Koordination und Kooperation auf zwei Stufen}

Im kooperationstheoretischen Teil wird knapp erörtert, wie es zur Durchsetzung einer Branchenvereinbarung durch Sozialstandards auf zwei Stufen kommen kann, die Verbände, große und kleine Unternehmen des Nordens als auch Unternehmen und Verbände des Südens, einschließt. ${ }^{4}$ Auf der ersten Stufe kooperieren die großen Marken-

3 Vgl. z. B. einen aktuellen Ratgeber des Instituts für Markt-Umwelt-Gesellschaft (Imug): http://www.csr-mittelstand.de/pdf/Schoenheit_CSR-Marketing_BIELEFELD_KOM_2008_02 _26.pdf (gel. am 31.01.2009).

$4 \quad$ Vgl. dazu ausführlich Biedermann 2007: 130-135 (Koordination der Marktführer) sowie 173-179 (Institutionalisierung von Branchenvereinbarungen). 
und Handelskonzerne, indem sie ihre Standards koordinieren. Auf der zweiten Stufe kooperieren die vorher koordinierten Marken- und Handelsunternehmen mit den Lieferanten und Produzenten des Südens, die jedoch erst durch eine umfassende Institutionalisierung ermöglicht wird. Ohne eine Kooperation auf der ersten Stufe wird die Kooperation zwischen einzelnen Marken- und Handelsunternehmen und den Lieferanten in Ländern des Südens instabil bleiben und lediglich in einzelnen Ausnahmefällen erfolgreich sein (z. B. Konzerne, die exklusive Vertragslieferanten haben, die nicht für andere Unternehmen produzieren).

Kooperation auf der ersten Stufe der dominierenden Marktführer bezeichnet eine Übereinkunft, Standards zu konvergieren und zu koordinieren oder gegebenenfalls gegenseitig anzuerkennen. Koordination der Standards im Sinne der Spieltheorie bedeutet, dass Konzerne und Unternehmen des Nordens keine grundsätzlichen, schwerwiegenden Widersprüche in ihren Standards haben, aber hohe Anreize einer Harmonisierung bestehen. Für alle Akteure ist eine Einigung auf einen einheitlichen „Satz“ an Standards deutlich sinnvoller, als wenn jedes Unternehmen seinen eigenen Ansatz verfolgt. Kooperationsanreize bei Sozialstandards bestehen, weil Konflikte negative Auswirkungen haben, und Koordinationsgewinne hohen Kollektivnutzen zu geringen Kosten stiften (vgl. Biedermann 2007: 131). Während zu Beginn der 1990er jedes Großunternehmen seine eigenen Standards bei Lieferanten durchsetzen wollte, hat sich dies als unmöglich erwiesen, sobald Lieferanten mehrere Markenfirmen mit je unterschiedlichen Standards beliefern. Koordination wird auch begünstigt, wenn und weil sich Standards im Laufe der Jahre immer ähnlicher geworden sind, z. B. durch ähnliche Erfahrungen, gleiche gesellschaftliche Erwartungen und unternehmensübergreifende Kommunikation, z. B. in Verbänden. ${ }^{5}$ Über diese inhaltliche Annäherung sind sich Praktiker als auch Wissenschaftler weitgehend einig (vgl. Rivoli 2003; Biedermann 2007; Krueger 2008). Die Qualität der Kooperation durch Koordination bei Sozialstandards ist vergleichbar mit der Abstimmung von Flugzeugrouten im Luftverkehr, der Verkehresregelung, z. B. an der Straßenampel (vgl. Stein 1982) oder der Belegung von Funkfrequenzen (vgl. Brams 1994). Eine Koordination ist im Sinne aller, es gibt keine besonderen Präferenzen, wodurch ein Verteilungskampf weitgehend vermieden werden kann und jedes Verhandlungsergebnis wesentlich besser ist, als eine Kollision. Übertragen auf die Praxis bei Sozialstandards in globalen Lieferketten bedeutet dies, dass es günstiger ist, Standardsätze zu koordinieren (wie z. B. Farben der Bodenmarkierungen zu Notausgängen, Aufhängehöhe und Anzahl von Feuerlöschern, Personenanzahl in Schlafunterkünften etc.), als wenn sich Kodizes, die z. T. mehr als hundert Bestimmungen enthalten können, widersprechen. Eine Koordination spart allen Beteiligten Kosten und erleichtert den Lieferanten die Einhaltung der Standards, wodurch eine Koordination attraktiv wird. Da sich Auftraggeber in einer interdependenten Situation wiederfinden, wenn sie Überschneidungen bei Lieferanten haben, entstehen Anreize, Standards zu koordinieren.

5 Ähnliche Prozesse gibt es in der internationalen Politik in der Umweltpolitik, in der eine zunehmende „cross-national policy convergence“ festgestellt wurde. Als entscheidende Variablen dafür wurden insbesondere die internationale Harmonisierung, transnationale Kommunikation, der Regulationswettbewerb sowie spezifische Problemlagen untersucht (Holzinger et al. 2008: 556-563). 
Gruppengrößen können erklären, warum Koordination im Norden einer Kooperation der Unternehmen im Süden sowie einer Kooperation der Markenfirmen mit den Lieferanten zwingend vorausgehen muss. Denn unabhängig von Macht- oder Größenverhältnissen der Unternehmen ist es allein schon kooperationstheoretisch wesentlich leichter, eine Branchenvereinbarung im Norden auf den Weg zu bringen, als im Süden, da die Produzenten im Süden eine ungleich größere Gruppe darstellen. So besteht in der sozialwissenschaftlichen Literatur Einigkeit, dass kleine Gruppen im einstelligen oder niedrigen zweistelligen Bereich wesentlich leichter zu gemeinsamen Beschlüssen kommen können, als große Gruppen mit Hundert und mehr Akteuren (vgl. Olson 1992; Hardin 1982; Spar 1994). Zwar befinden sich in einer globalen Branche, insbesondere im überwiegend mittelständisch geprägten Konsumgüterbereich, weltweit beinahe zahllos viele Akteure, die unabhängig voneinander agieren können. Da die privaten Akteure jedoch zweifelsohne in großem Maße unterschiedlich einflussreich sind, kann von Hierarchie zwischen Marktakteuren, insbesondere zwischen Markenunternehmen auf der einen Seite und Fabriken im Süden auf der anderen Seite, ausgegangen werden. Branchenvereinbarungen, die von relativ wenigen, aber marktbeherrschenden Unternehmen angestoßen und aufrecht gehalten werden, könnten so wie ethische Kartelle wirken, wenn Produzenten, die bestimmte Marktregeln nicht beachten, mit ihren Produkten ausgeschlossen oder z. B. nur noch Marktnischen bedienen können. Weiterhin können sich die wenigen Marktführer gegenseitig besser kontrollieren, sodass Zusagen zur gemeinsamen Einhaltung von Standards glaubwürdig werden (credible commitments in der Kooperationsliteratur).

Fehlt hingegen die Koordinierung durch Marktführer, rückt die Durchsetzung von Branchenstandards in weite Ferne. Denn die Produzenten im Süden sind praktisch nicht kooperationsfähig, da sie viel zu zahlreich sind und ihre möglichen Zusagen untereinander kaum gegenseitig kontrollieren können. Deshalb bestehen hier auch keine Anreize, Standards von sich aus zu erhöhen bzw. einzuhalten. Wenn jedoch ein großer Teil des Marktes (faktisch die Abnehmer) seine Standards koordiniert hat, so bestehen auch Anreize im Süden, die koordinierten Standards zu beachten, um die Option auf den freien Marktzugang zu erhalten bzw. zu erreichen. Die Qualität der Kooperation ist hier jedoch anders als die innerhalb der Marktführergruppe. Lieferanten im Süden müssen von ihren Abnehmern für die Kooperation durch gemeinsam erhöhte Standards belohnt werden, und zwar durch stabile Lieferbeziehungen sowie Bereitschaft, höhere Preise in Kauf zu nehmen. Spieltheoretisch kann diese Form der Kooperation als „Tit for Tat“ bezeichnet werden (vgl. Axelrod 1995). Kooperation kann nur dann entstehen und dauerhaft aufrecht erhalten werden, wenn ein Ende der Kooperation nicht abzusehen ist, Kooperation den Akteuren Nutzen bringt während Nichtkooperation wechselseitig geahndet bzw. bestraft wird, was im Allgemeinen durch eine umfassende Institutionalisierung begünstigt wird. Wenn Unternehmen des Südens nicht kooperieren, droht ihnen Auftragsentzug und schließlich der Niedergang der Firma. Wenn Abnehmer im Norden nicht kooperieren (stabile Lieferbeziehungen, Akzeptanz eines höheren Preises), bestehen für Lieferanten keine Anreize, die Standards einzuhalten. Kooperation auf der zweiten Stufe ist somit durch gegenseitige Belohnung, aber auch durch glaubhafte Abschreckungen (believable threats in der Kooperationsliteratur) gekennzeichnet. 
Neben den Branchenverbänden kommen bei einer Institutionalisierung wiederum die großen Marktführer infrage, die schon Erfahrung mit ihren unilateralen Programmen gemacht haben. Ganz praktisch betrifft dies die Finanzierung des Systems, sei es durch personelle Ausstattung und Beratung oder durch die finanzielle Ausstattung bzw. Initialzündung. ${ }^{6}$ Die Einhaltung der Standards müsste unabhängig von Dritten, z. B. sogenannten Monitoring-Agenturen (oder zusätzlich Gewerkschaften, NGOs), überprüft werden, die ihrerseits einem spezifischen Training in der Branche unterzogen werden, um vergleichbare und wiederholbare Ergebnisse abliefern zu können. Denn Glaubwürdigkeit und Vertrauen untereinander, ein wesentliches zu erarbeitendes Kapital von Branchenstandards, kann nicht entstehen, wenn jedes Unternehmen nach eigener Methodik die Standards überprüft und nur deren Einhaltung vorgibt.

Wenn sich marktführende Unternehmen zu Branchenstandards bekennen und einen institutionellen Rahmen unterstützen oder bereitstellen, Branchenstandards durchzusetzen, dann kann eine Branchenvereinbarung zum Erfolg führen. Die Koordinierung der Standards auf der ersten Stufe der Marktführer durch die kooperationstheoretisch ein kollektiver Hegemon entstehen kann, ist somit eine zwingende Voraussetzung für die Durchsetzung durch Kooperation der Lieferanten auf der zweiten Stufe. Die Kooperation auf der ersten Stufe ist ein Koordinationsspiel, während die umfassende Kooperation auf der zweiten Stufe aufgrund geringerer Anreize schwerer zu erreichen ist und einer Institutionalisierung bedarf um Zusagen Glaubwürdigkeit zu verleihen, sowie nachvollziehbare, glaubhafte Abschreckungen durch Auftragsentzug zu ermöglichen.

\section{Die Spielzeugindustrie: Institutionalisierung der Branchenvereinbarung}

\subsection{Der globale Spielzeugmarkt und Sozialstandards}

In China, hauptsächlich verteilt in der Provinz Guangdong (Kanton),der Region um Shenzhen, gibt es zwischen 6000 und 10000 Spielzeug herstellende Fabriken, bei denen in der Hauptsaison bis zu drei Millionen Menschen beschäftigt sind (vgl. Chan 2003: 44). Noch Anfang der 1990er Jahre waren es erst 1700 Betriebe. Mit etwa 7,5 Milliarden Euro Exportwert führt China mehr Spielwaren aus, als in Europa und den USA zusammen hergestellt werden. 70 bis 80 Prozent des weltweit verkauften Spielzeugs kommt heute aus China (vgl. ICTI 2008). Der weltweite Spielzeugmarkt wird vom Weltverband mit einem Wert von 72 Milliarden US-Dollar angegeben, mit positiven Zukunftsaussichten (vgl. Guinaudeau 2008: 20). Doch die Phase des schnellen Wachstums in China ist vorbei, auch Unternehmenspleiten gehören seit Jahren zur

6 Die hegemoniale Stabilitätstheorie nach Charles Kindleberger (1973) betrachtet die Hegemonialstellung einzelner Akteureü als eine wichtige Voraussetzung für die Stabilität und Institutionalisierung internationaler Kooperation zur Aufrechterhaltung von Finanzsystemen oder sicherheitspolitischen Zielsetzungen etc. Fehlt ein überragender Hegemon oder ein kollektiver Hegemon, sind internationale Systeme latent instabil. Gleiches kann für Private Governance durch Sozialstandards in globalen Lieferketten geltend gemacht werden. 
Normalität. ${ }^{7}$ Höhere Rohstoffkosten sowie gestiegene Löhne in anderen Branchen und Regionen Chinas verstärken den Kostendruck. Die Produzenten müssen mit harten Bandagen um Zugang zu den großen westlichen Vertriebskanälen konkurrieren, viele geben Aufträge auch an Subunternehmen weiter, um Standards leichter zu umgehen und um billiger produzieren zu können. Selbst Kinderarbeit tritt derzeit wieder in der Branche auf, obwohl diese selbst von NGOs lange Zeit nicht als ein nennenswertes Problem in der Branche identifiziert wurde. ${ }^{8}$ Der Wettbewerb der großen Markenunternehmen und des Handels wird seit Jahren verschärft auf dem Rücken der OEM-Hersteller ${ }^{9}$ in China ausgetragen, die über stark gesunkene Preise und die Auftraggeber klagen, die nicht mehr wie in den 1970er und 80er Jahren stabile Lieferantenbeziehungen anstreben würden, sondern den gerade günstigsten und schnellsten Lieferanten aussuchen. ${ }^{10} \mathrm{Um}$ auf aktuelle Modetrends reagieren zu können, wurden die Lieferfristen extrem verkürzt. Produzenten in China müssen zudem die stark im Preis gestiegenen Rohstoffe (v. a. Plastik), die den Großteil der Produktionskosten ausmachen, „,vorschießen“. Die prekäre Situation der Produzenten hat in den letzten Jahren zur paradoxen Situation geführt, dass den Spielzeugfirmen die Arbeiter wegblieben, obwohl angenommen wird, dass China über eine verdeckte Arbeitslosigkeit von 200 Millionen Menschen verfügt und jedes Jahr mehr als 100 Millionen Wanderarbeiter in die Küstenstädte strömen. Da die Hälfte des Jahresumsatzes zur Vorweihnachtszeit gemacht wird, findet die Produktion hauptsächlich von Juli bis September statt, mit den entsprechenden Auswirkungen auf Arbeitszeiten und den Arbeitsschutz.

Der rasanten Zunahme an Fabriken in China steht ein Konzentrationsprozess in den wichtigsten Spielzeugmärkten, den USA und Europa, gegenüber. Während die Spielzeugindustrie eine klassische mittelständische Branche mit immer noch hunderten von Herstellern in den Industrieländern ist, sind es dennoch die Global Player und Aktiengesellschaften in Vermarktung und Vertrieb, die maßgeblich die Dynamik bestimmen. Die größten Markenhersteller Mattel (2006: 5 Mrd. US-Dollar Umsatz) und Hasbro (3,2 Mrd. US-Dollar Umsatz), die wie die meisten Markenunternehmen vor allem als OEM-Auftraggeber in Erscheinung treten, haben in den letzten Jahren zahlreiche Branchengrößen übernommen. Auch regionale Branchenführer wie Smoby (größter Hersteller Frankreichs) oder Simba-Dickie in Deutschland haben in den letzten Jahren zahlreiche Unternehmen übernommen. Die jüngste Übernahme von Smoby durch Simba-Dickie im März 2008 bestätigt, dass auch zukünftig ein weiterer Konzentrati-

7 Interview mit Monina Wong vom Hong Kong Christian Industrial Committe (HKIC) am 23.05.2003 in Berlin (Biedermann 2007: 114).

8 In einer Presseerklärung aus dem Jahr 2008 weist der deutsche Spielwarenhersteller Simba-Dickie darauf hin, dass aufgrund des unerwarteten Arbeitskräftemangels wieder Kinderarbeit bei chinesischen Spielzeugfirmen auftritt, allerdings nicht bei Lieferanten von Simba-Dickie, wo solche Fälle aufgrund der engen Beziehungen zu den Joint Venture Betrieben ausgeschlossen werden können (vgl. http://simba-dickie-group.de/de/aktuelles/nachrichten/kampf_gegen_kinderarbeit /seite_1.shtml).

$9 \quad$ OEM=Original Equipment Manufacturing: die Produktionsvergabe eigener Unternehmensprodukte an Fremdproduzenten.

10 Interview mit Monina Wong am 23.05.2003 in Berlin (Biedermann 2007: 114). 
onsprozess in der Branche zu erwarten ist. ${ }^{11}$ Mittlerweile können etwa 50 Markenunternehmen 90 Prozent des amerikanischen Spielzeugmarkts kontrollieren. In Deutschland entfallen auf die 30 größten Markenunternehmen 80 Prozent des Marktes (vgl. Kleinert 2004).

Noch konzentrierter stellt sich der Vertrieb der Spielwaren dar, der im Wesentlichen dreigliedrig ist: Zum einen Spielzeugketten wie Toys 'R'Us und Chicco, dann große Einzelhandelsketten wie Walmart und Target in den USA, Argos in Großbritannien, Carrefour in Frankreich oder KarstadtQuelle in Deutschland. Schließlich Einzelhandelseinkaufsverbände wie vedes oder Idee\&Spiel. Walmart, Target und Toys'R'Us vertreiben zusammen über 50 Prozent des in den USA gehandelten Spielzeugs. Toys'R'Us, der weltgrößte Spielwareneinzelhändler, hat in den letzten Jahrzehnten zahlreiche andere Einzelhandelsketten in den Ruin getrieben und muss sich nun selbst umstrukturieren, um im Preiskampf mit Walmart (mittlerweile der größte Spielwarenhändler der Welt) zu bestehen. Um die Abhängigkeit von den großen Händlern zu verringern, hat der Weltmarktführer Mattel auch den Direkt-Onlineverkauf seiner Produkte als neuen Vertriebsweg über seine internationalen Homepages erschlossen. Dadurch, dass Markenspielzeug eine hohe Reputation und damit eine entsprechende Nachfrage genießt, können die Händler nicht auf dieses Renommee verzichten, so dass sich eine gewisse gegenseitige Abhängigkeit und Kooperation ergibt. So räumen z. B. Händler im Gegenzug für exklusive Vertriebsrechte bestimmter Spielzeuge den Markenunternehmen besondere Regalplätze ein. Dennoch haben die wichtigsten Handelsunternehmen wie Walmart, Toys' ${ }^{\prime}$ Us oder KartstadtQuelle ihre eigenen Spielzeugentwickler und in den letzten Jahren den Anteil an Eigenmarken sukzessive erhöht, um eine höhere Handelsspanne zu erzielen. Walmart allein setzt mehr als 12 Mrd. US-Dollar mit Spielwaren um, was die eindrucksvolle Position des führenden Handelskonzerns bestätigt. Allein in China hat Walmart 5000 Lieferanten für Konsumgüter (davon 4400 in Kanton), die zu 70\% von dort bezogen werden (vgl. Biedermann 2007: 155).

Seit den 1990er Jahren haben Nichtregierungsorganisationen (NGOs) die Arbeitsbedingungen in der chinesischen Spielzeugindustrie öffentlichkeitswirksam angeprangert, die Zusammenhänge in der Branche analysiert und die Hauptverantwortlichkeit der westlichen Markenunternehmen artikuliert. ${ }^{12}$ Wie zu Beginn der Auseinandersetzung über die Arbeitsbedingungen in Entwicklungs- und Schwellenländern üblich, wiesen die Global Player die Verantwortung für die Arbeitsbedingungen bei den Auftragnehmern weit von sich. Umso stärker haben sich in der Folge NGOs bemüht, die Verantwortlichkeit der westlichen Markenkonzerne herauszuarbeiten, indem sie auf den Preisdruck, die immer kürzeren Lieferzeiten, vermehrten Druck auf Zulieferer durch unstete Lieferantenbeziehungen, sowie auf die extrem hohen Arbeitszeiten mit 80

11 Vgl. http://simba-dickie-group.de/de/aktuelles/nachrichten/spielwarenhersteller_simba_dickie_ group_uebernimmt_franzoesische_smoby_gruppe.shtml (gel. am 30.01.2009).

121993 kam es zu verheerenden Fabrikbränden in Thailand (Bangkok) und China (Shenzhen), bei denen hunderte von Menschen zum Teil schwer verletzt wurden und viele ihr Leben lassen mussten. The Hong Kong Toy Coalition war die erste Initiative von Nichtregierungsorganisationen, die 1995 den ersten Bericht über die Arbeitsbedingungen in der Spielzeugindustrie anfertigten (Biedermann 2007: 99). 
Wochenstunden und weit mehr verwiesen, oder indem sie eindrucksvolle Produktionskostenbeispiele die zigfach höheren Handelspreise gegenüber stellten, um die Machtverhältnisse zu verdeutlichen (vgl. AMRC 1998; Piepel 2001; Heidel et al. 2002). Die Zustände in den Fabriken wurden in detaillierten Berichten erörtert (vgl. HKCIC 2000, 2001) und wurden von westlichen Brancheninsidern durchaus bestätigt. NGOs aus Hongkong sind seit Mitte der 1990er Jahre auf NGOs in Europa und den USA zugegangen, um die Kampagne für „fair“ produziertes Spielzeug auch in den Norden zu tragen, wo die großen Spielzeugkonzerne sitzen. In verschiedenen europäischen Ländern wie England, Frankreich, Deutschland, die Schweiz oder Schweden sowie den USA formierten sich zivile Gruppen aus dem kirchlichen oder globalisierungskritischen NGO-Sektor, die z. T. bis heute noch aktiv mit dem Thema befasst sind. Gewerkschaften haben in der Kampagne keine nennenswerte Rolle gespielt. Bis Ende der 1990er Jahre hat sich praktisch jedes wichtige Marken- und Handelsunternehmen einen eigenen Verhaltenskodex zur Sicherung der Arbeitsbedingungen im Süden gegeben (vgl. Biedermann 2007: 138).

Aufgrund dieser Wettbewerbssituation schätzt der Hongkong-chinesische Spielzeugverband (Hong Kong Toys Council: HKTC) seinen eigenen Einfluss auf die Lieferanten als sehr gering ein, Standards zu unterstützen. ${ }^{13}$ Gesprächspartner des Verfassers von Verbänden und der Industrie vertreten die Auffassung, dass erst das Engagement der Marktführer dazu führen kann, dass Produzenten in China sowie die vielen kleineren Auftraggeber Standards verstärkt berücksichtigen. ${ }^{14}$ Auch gruppentheoretisch würde man hier eindeutig zum Schluss kommen, dass Kooperation unter den vielen tausend Lieferanten durch eine Branchenvereinbarung in China unmöglich ist, wenn es kein Monopol (den Staat) oder andere Akteure von außen gibt, die hier eingreifen.

\subsection{Kooperation der Marktführer und Institutionalisierung der Branchenver- einbarung}

Obwohl es bereits seit 1995 einen Branchenkodex in der Spielzeugindustrie gibt, haben nur die wenigsten Unternehmen diesen Kodex wirklich unterstützt bzw. in den eigenen und in den Zulieferfabriken angewendet. Der Branchenkodex wurde seinerzeit auf Betreiben des britischen Spielwarenverbands und seines Präsidenten David Hawtin maßgeblich entwickelt, nachdem die britische Spielzeugindustrie aufgrund verheerender Unfälle in China (Hongkong war damals noch britische Kronkolonie) stark durch NGOs kritisiert wurde. Die Standards wurden auch vom Weltverband International Council of the Toy Industries (ICTI) übernommen, ohne allerdings wirklichen Einfluss in der Branche zu entwickeln. Ein Grund dafür ist, dass sämtliche Branchenführer (bis auf Hasbro, der zweitgrößte Spielwarenproduzent der Welt, der

13 Interview mit Fong Chi-vai und Ng Kwok-keung vom Hongkong Toys Council (HKTC) am 06.10. 2004 in Hongkong (Biedermann 2007).

14 Interviews mit Christian Ewert (Vizepräsident von ICTI und CEO des ICTI-Care-Prozesses (18.04.2005) in München, mit Kelvin Ho, Regionaldirektor von CSCC (California Safety Compliance Corporation) am 04.10.2004 in Hongkong, Andreas Schmitt und Ekkehard Babion von Simba-Dickie am 21.02.2005 in Fürth sowie den Verbandsrepräsentanten von HKTC (Biedermann 2007). 
maßgeblich an der Weiterentwicklung des Kodexes im Rahmen des amerikanischen Verbands beteiligt war) ihren eigenen Kodex anwendeten, ebenso wie Disney, Mattel oder McDonald's. Ein weiterer, dass der Kodex lediglich auf dem Papier existierte, ohne jedwede Institutionalisierung vor Ort. Die anspruchsvollsten Standards, auch sektorübergreifend, hat der Weltmarktführer Mattel mit seinen Global Manufacturing Principles (GMP) ${ }^{15}$ auf den Weg gebracht, was sich bei Mindestlöhnen und Arbeitszeiten ausdrückt. Dies aber auch insofern, als er nicht nur den Standardkatalog der bekannten Kriterien der Internationalen Arbeitsorganisation (ILO) zur Nichtdiskriminierung, Kinderarbeitsverbot, Gewerkschaftsfreiheit etc. enthält, sondern auch umfassende Vorschriften zum Arbeitsschutz mit vielen industriespezifischen Bestimmungen. Die Standards werden zudem von einem unabhängigen Gremium aus Wissenschaftlern geprüft. Mattel stellt die Ergebnisse der Überprüfungen auf seiner Webseite aus. ${ }^{16}$ NGOs kritisieren Mattel jedoch, dass die Standards vor allem bei den firmeneigenen Lieferanten (12 Produktionsstätten in Asien) beachtet werden, während bei 77 weiteren Vertragslieferanten die Durchsetzung weniger überzeugend gelinge (vgl. Biedermann 2007: 143-148). Das einzige größere Unternehmen in Deutschland, das von Beginn die Branchenstandards anerkannt und tatsächlich angewendet hat, war Zapf-Creation, der bekannte fränkische Puppenhersteller, der in den 1990er Jahren einen großen Aufschwung erlebte. ${ }^{17}$ Weder in den USA oder Europa gab es eine nennenswerte Bewegung, die Branchenstandards zu unterstützen. Neben der fehlenden Unterstützung der Marktführer war dafür in zweiter Linie auch die fehlende Institutionalisierung verantwortlich (so argumentierte z. B. Simba-Dickie). Es gab keine allgemeingültige Methode, die Standards zu überprüfen und sie durchzusetzen. Sie waren eine hektische Antwort auf die zivilen Proteste in den westlichen Industriestaaten und existierten vor allem auf dem Papier. Für die Industrie hat der Branchenkodex zwischen 1995 und 2003 somit keine Bedeutung erlangt.

Wie sah es im Handel aus, nachdem auch die großen Handelsunternehmen Walmart und Toys' R'Us in den USA, aber auch europäische Unternehmen wie KarstadtQuelle, zivilen Protesten in den 1990er Jahren zuvorderst begegnen mussten? Walmart hat einen unilateralen Ansatz gewählt, während Toys' ${ }^{\prime}$ 'Us sehr eng mit der Gruppeninitiative Social Accountability International, die den anspruchsvollen SA8000-Standard auf den Weg gebracht hat, verhaftet ist. Weitere Branchenführer aus dem Handel sowie mittelgroße Unternehmen haben weitere, je eigene Verhaltenskodizes auf den Weg gebracht. Letztlich hat dies zu einer unüberschaubaren Anzahl an Verhaltenskodizes in der Spielzeugindustrie geführt, bei der die Fabriken in China völlig überfordert wurden und z. T. 70 verschiedene Verhaltenskodizes und mehr beachten mussten, mit entsprechend häufigen Kontrollen. ${ }^{18}$ Selbst für Mattel wurde es so unmöglich, die eigenen Standards in Fabriken durchzusetzen, die auch andere Marktführer wie

15 Vgl. ausführlich Biedermann (2007: 143-148).

16 Vgl. www.mattel.com/about_us/Corp_Responsibility/cr_mimco.asp (gel. am 31.01.2009).

Christian Ewert, seinerzeit für die Implementierung des Branchenkodexes bei Zapf-Creation verantwortlich, ist heute Präsident der ICTI Care Foundation.

18 Interview mit Maren Böhm, Leiterin Gesellschaftspolitik bei KarstadtQuelle am 23.02.2005 in Essen. 
Hasbro und Disney beliefern. Die Interdependenz verstärkte somit den Wunsch auch der Großabnehmer nach einheitlichen Standards, nachdem über mehrere Jahre bis 2003 unilateralen Ansätzen der Vorzug gegeben wurde. Ein Vergleich der Standards hat ergeben, dass sie sich stark ähneln (vgl. Biedermann 2007: 153 und 167).

2004 hat sich die Situation geändert, als Mattel eingestehen musste, dass es seinen anspruchsvollen Kodex gerade in jenen Fabriken, die auch für andere Unternehmen produzieren, nicht überzeugend durchsetzen konnte. ${ }^{19}$ Mit Hasbro, dem größten Konkurrenten, sprach sich Mattel im US-amerikanischen Industrieverband ab, künftig den Branchenkodex zu propagieren und stark zu unterstützen: „If Hasbro and Walmart would enforce the same standards vigorously, Mattel would have an easier time convincing suppliers to reduce excessive hours" (gemeint ist die Anzahl der Arbeitsstunden: der Verfasser) ${ }^{20}$. Mattel gab seine eigenen Standards nicht auf, fährt aber seitdem eine Doppelstrategie, um auch in Fabriken Standards durchzusetzen, die für andere Firmen produzieren. Bereits im Februar 2004 erklärte der Weltverband ICTI in einer Presseerklärung, dass aus allen Sektoren der Spielzeugbranche - Markennamen, Produzenten, Händler und Lizenznehmer - Unterstützung für die Brancheninitiative kommt. Die Initiative wird von den größten Industrieunternehmen der Branche, Mattel, Hasbro, Leapfrog, Lego und weiteren Branchenführern wie z. B. Maisto und Bandai aus Japan, Zapf-Creation, Playmobil oder Simba-Dickie aus Deutschland, unterstützt. Der amerikanische Verband machte bereits im Herbst 2005 die Mitgliedschaft im Verband von der Unterstützung der Date Certain-Kampagne verantwortlich, der zufolge Mitgliedsunternehmen ein verbindliches Datum angeben müssen, $a b$ dem sie nur noch von ICTIzertifizierten Unternehmen kaufen. Nach Alan Hassenfeld, Firmenchef von Hasbro und Co-Vorsitzender der ICTI Care Foundation, würden Sweatshops in der Spielzeugindustrie bald der Vergangenheit angehören. ${ }^{21}$

Der Branchenkodex der Spielzeugindustrie verbietet Kinderarbeit (unter 14 Jahre), Zwangsarbeit sowie Gefangenenarbeit und Diskriminierung am Arbeitsplatz, womit er diejenigen Kernforderungen der ILO erfüllt, die auch für Unternehmensverhaltenskodizes selbstverständlich sind. Die Arbeitsstunden richten sich wie bei den Unternehmenskodizes nach den jeweiligen gesetzlichen Vorgaben, oder, falls es solche nicht gibt, nach sozialen, menschlichen und produktiven Gesichtspunkten. Den Arbeitern steht ein freier Tag pro Woche zu. Generell sind Überstunden freiwillig, wobei in der Realität Arbeiter Überstunden machen, um ihre Niedriglöhne aufzubessern. Die Be-

19 "The majority of suppliers manufacturing products for Mattel are also producing for other companies, both within and outside the industry. Because of the existence of multiple codes of business conduct, it is difficult for employees at these factories to be fully informed concerning each company's individual code. (...), this is one of the main reasons that Mattel fully supports the implementation of an industry-wide code of business conduct for the toy industry (...)." Von der Mattel-Homepage, zitiert nach Biedermann (2007: 148).

20 Los Angeles Times, 26. November 2004. Prakash Sethi, Vorsitzender des Kontrollgremiums der GMP-Principles von Mattel, musste zudem eingestehen: „It is hard for Mattel to impose conditions unilaterally when a vendor is operating with other toy companies, since other buyers do not place the same emphasis on compliance as Mattel." In: Corporate Social Issue Reporter, December 2004, 9.

21 „Sweatshops are going to become history“, vgl. Mainland Toy Companies facing the ICTI Dilemma, http://www.laborrights.org/creating-a-sweatfree-world/544 (gel. am 15.01.2009). 
zahlung richtet sich nach dem lokalen Mindestlohn, der innerhalb Chinas von Provinz zu Provinz auf einem niedrigen Niveau abweichen und auch lokal gestaffelt sein kann und nicht unterschritten werden darf. Überstunden müssen bezahlt werden. Disziplinarische Maßnahmen, die in chinesischen Betrieben eher die Regel als die Ausnahme darstellen, sind untersagt, ebenso wie von den Arbeitern zu tragende Gebühren für Arbeitsschutzmaßnahmen. Der Kodex sieht Arbeitnehmervertretung im gesetzlich erlaubten Rahmen vor: Passagen im konkretisierten Guidance Document könnten mit gutem Willen dahingehend ausgelegt werden, ebenso Arbeitnehmervertretung vorzuschreiben, wie dies etwa beim SA8000-Standard (,parallel means of association and bargaining") der Fall ist, wenn es heißt: "Various ways in which employees could approach management include trade unions, employee committees (grievance, safety, etc.), or employee selected representatives, regular meetings, etc." (ICTI COBP 2004: 3522). Doch gibt auch Christian Ewert (CEO des CARE ${ }^{23}$-Prozesses, Vizepräsident des Weltverbands) zu, dass die Arbeiterrechte Kollektivverhandlungen und Vereinigungsfreiheit ,noch einmal eine andere Qualität baben" (Biedermann 2007: 184) als die entsprechenden Klauseln im ICTI-Code. Besonders ausgearbeitet sind die Vorschriften zur Unterkunft, zu hygienischen Einrichtungen für die Arbeiter, zu den Maßnahmen zum Feuerschutz und zum Umgang mit gefährlichen Chemikalien sowie generell zum Sicherheits- und Gesundheitsschutz der Arbeiter. Das Monitoring des Kodex wird ausschließlich durch unabhängige Monitoringagenturen durchgeführt, die in einem fünfmonatigen Auswahlprozess durch ein Expertengremium einer Testreihe unterzogen wurden, bei dem schließlich sechs Unternehmen übrig blieben, die exklusiv die Fabriken auf die Einhaltung des ICTIKodex prüfen. Ergebnisse werden mit den Fabrikbesitzern besprochen, die einen spezifischen Corrective Action Plan für das Unternehmen zufolge haben, der in einem zweiten oder gar dritten Audit auf Umsetzung überprüft wird. Werden die Maßnahmen nicht umgesetzt, wird von Auftraggebern verlangt, die Lieferbeziehungen zu beenden.

Von Markenunternehmen verlangt der Verband eine verbindliche Zusage, bis wann sie ihre Lieferanten auf den ICTI-Kodex zertifiziert haben. Fabriken im Süden, die zertifiziert worden sind, bekommen das sogenannte Seal of Compliance. Außerdem wird der Fabrikname auf der Homepage des Weltverbands veröffentlicht, samt Standortregion, Mitarbeiterzahl, sowie Dauer der Gültigkeit des Zertifikats. Eine weitere Datenbank enthält die Namen der Markenunternehmen (also der Auftraggeber), die sich im Rahmen der Verpflichtungserklärung (Date Certain Campaign) zum ICTI-Kodex bekannt haben und die Zertifikate ihrer Lieferanten vorweisen müssen.

Zwischen 2004 und 2005 haben sich ca. 200 Markenunternehmen dazu bekannt, ihre Lieferanten bis zum 1. Januar 2006 zu zertifizieren. Nach Verbandsaussagen hatten sich damit über 60 Prozent des Gesamtumsatzes in den USA verpflichtet, in Europa bereits über 70 Prozent. Weltweit wurden durch die Verpflichtungserklärungen durch den Spielwarenhandel zudem über 50 Prozent des Spielzeugmarkts abgedeckt. Zum

\footnotetext{
22 Das Handbuch zum CARE-Prozess kann Online eingesehen werden: http://www.icticare.org/handbook/section1.html (gel. am 31.03.2008).

23 Caring, Awareness, Responsibility, Ethics (CARE): Damit bezeichnet ICTI den Gesamtprozess, Sozialstandards in der globalen Spielzeugindustrie einzuführen.
} 
Februar 2005 waren in ICTI-zertifizierten Betrieben in China etwa 200000 Menschen beschäftigt, womit die Vorstellungen der Branche übertroffen wurden. 155 Audits wurden bis dahin durchgeführt und ca. 100 Zertifikate (April 2005) ausgestellt. Drei Jahre später gibt die Homepage des ICTI-CARE-Prozesses im Februar 2008 an, dass 747 Fabriken das Seal of Compliance haben, während insgesamt 1461 Fabriken zur Prüfung registriert sind, was etwa einer Million Arbeitnehmern zugute käme. ${ }^{24} \mathrm{Im} \mathrm{Jahr}$ 2007 wurden 1800 Audits in 800 Spielzeugfabriken durchgeführt, in deren Folge es zur Schließung von acht Fabriken kam, nachdem eine systematische und wiederholte Verletzung der Standards festgestellt wurde, weitere 33 Fabriken wurden unter Probe gesetzt und müssen sich dem Corrective Action Plan unterziehen (vgl. ICTI 2008: 16).

Auch der Deutsche Verband der Spielzeugindustrie (DVSI), lange Zeit blass geblieben, hat sich seit 2005 dazu entschlossen, stärker auf seine Mitgliedsunternehmen in Deutschland einzuwirken. So werden auf der Homepage alle Unternehmen mit einem Ampelsystem aufgeführt, aus dem hervorgeht, wie die Unternehmen zum Branchenkodex stehen. Auf den DVSI und seine Mitgliedsunternehmen ist zuvor erheblicher Druck durch KarstadtQuelle und die Außenhandelsvereinigung des deutschen Einzelhandels (AVE) ausgeübt worden, endlich Sozialstandards in den Fabriken zu beachten, andernfalls werde von bestimmten Lieferanten nicht länger bezogen. Der DVSI hilft zudem seinen Mitgliedsunternehmen, Informationen über Lieferanten in China zu bekommen und den Zertifizierungsprozess zu organisieren. ${ }^{25}$ Zum ersten Januar 2009 ist die Mitgliedschaft im DVSI an den Nachweis gebunden, dass sich Mitgliedsunternehmen dem Date Certain-Prozess angeschlossen haben. Spätestens bis zum 31.12.2008 mussten sich nun die Mitgliedsunternehmen diesbezüglich erklären. ${ }^{26}$

Dies bedeutet, dass Unternehmen angeben müssen, ab welchem Zeitpunkt sie nur noch von zertifizierten Lieferanten Waren beziehen. ${ }^{27}$ Den Listenansatz hat der DVSI von der Aktion fair spielt, der deutschen Kampagne für „faires“ Spielzeug (vor allem Misereor, Werkstatt Ökonomie) übernommen, die über viele Jahre gedrängt hat, Sozialstandards zu beachten und die, mit anderen NGOs in Industrieländern und Hong Kong, einen maßgeblichen Beitrag geleistet hat, dass Dynamik in die Branche gekommen ist. ${ }^{28}$ In einer Presseerklärung zur Spielwarenmesse in Nürnberg im Februar 2008 kritisiert die Aktion fair spielt, dass von den insgesamt 43 Herstellern, die mit der Umsetzung des ICTI-Kodexes bei den Zulieferern in China begonnen haben, lediglich

$24 \quad$ Vgl. www.icti-care.org/index.html (gel. am 31.01.2009).

25 Vgl. www.toy.de/news/index1760.html (gel. am 31.01.2009).

26 In dem Beschluss der Mitgliederversammlung vom 2./3. Juli 2008 heißt es wörtlich: „Jedes DVSI-Mitglied, das Produkte aus Schwellenländern, insbesondere aus China bezieht ist verpflichtet, spätestens bis zum 31.12.2008 verbindlich zu erklären, dass es für Spielzeuge am Date Certain Programm von ICTI teilnimmt oder anderweitig den Nachweis menschenwürdiger Arbeitsbedingungen führt. (...) Ein Verstoß gegen diese Verpflichtung ist ein Ausschlussgrund im Sinne des $\$ 4$ Ziff 3a) der Satzung des DVSI.““

27 Vgl. www.toy-icti.org/about/newsarticle.html (gel. am 4.01.2009): German Toy Association makes Date Certain a requirement for all members.

28 Diese Einschätzung des Autors wurde auch von KarstadtQuelle bestätigt. Ausführlich zu den Hintergründen und dem Einfluss der „fair spielt"-Kampagne von Misereor und anderen Akteuren siehe Biedermann (2007). 
15 bereits heute nur von ICTI zertifizierten Betrieben Waren beziehen. ${ }^{29}$ Von den DVSI-Mitgliedsunternehmen haben sich laut Homepage des DVSI (Stand Januar 2009) 80 Unternehmen zu Date Certain zum Januar 2009 bekannt, 27 Unternehmen gaben an, ausschließlich in Deutschland und Europa zu produzieren, während von 18 Unternehmen keine Reaktion kam. ${ }^{30}$ Es wird sich also zeigen, inwiefern der Druck vom DVSI zu weiteren Ergebnissen führt.

Seit 2004 führt der Weltspielwarenverband auch Verhandlungen mit Unternehmen aus dem Handel. Walmart und Toys' ${ }^{\prime}$ 'Us, zunächst skeptisch gegenüber der neuen Initiative, erkennen nun ebenfalls den Standard an und akzeptieren ihn wie die eigenen. Dies bedeutet, sie verlangen keine weiteren Überprüfungen mehr bei Lieferanten, wenn die über ein ICTI-Zertifikat verfügen. Das gleiche gilt für die europäische Business Social Compliance Initiative (BSCI), die von der Außenhandelsvereinigung des deutschen Einzelhandels (AVE) und weiteren europäischen Einzelhandelsunternehmen und Verbänden getragen wird. Durch die hohe Kongruenz, die die Standards aufweisen, wurden hier gegenseitige Einverständniserklärungen beschlossen („Memorandum of Understanding").

Die beschleunigte Ausbreitung in den ersten Jahren hat jedoch auch zu Kritik aus dem Handel geführt. Aufgrund eigener Erfahrungen im Rahmen der AVE und der BSCI sind Handelsunternehmen aufgrund der verdächtig schnellen Entwicklung bis 2006 skeptisch geworden. ICTI hat deshalb bereits Reformen eingeleitet: So bestimmt ICTI selbst, welches Monitoringunternehmen welche Fabrik prüft, um einer Kommerzialisierung des Systems vorzubeugen. Inspektionen werden nicht mehr von den Fabriken selbst in Auftrag gegeben, sondern von der ICTI Care Foundation, außerdem erscheinen die Kontrolleure künftig unangemeldet zur Inspektion, um Betrügerein zu erschweren (vgl. Kleinert 2008: 201). Das System wird insgesamt permanent überprüft und verbessert werden. So wurden 2007 auch erstmals zwei Vertreter von NGOs im Governance Board der ICTI Care Foundation aufgenommen. Weitere Audit-Firmen wurden akkreditiert, um mehr Kontrollen durchführen zu können (vgl. ICTI 2008: 8), die zudem künftig den Standards des International Register of Certified Auditors (IRCA) genügen müssen. Angesichts der ehrgeizigen Zielsetzung, die weltweit einmalig ist, wundert es auch nicht, dass es nach wie vor viele Berichte von NGOs (vgl. Kleinert 2008) aber auch Wissenschaftlern (vgl. Egels-Zandén 2007) gibt, die zahlreiche Mängel in der Durchsetzung des Branchenstandards feststellen. So wird z. B. kritisiert, dass die Lieferbeziehungen der Markenfirmen weiterhin nicht transparent sind, das Date Certain Programm letztlich freiwillig ist und nicht wirklich überprüft wird und somit ohne ernste Konsequenzen bleibt. ${ }^{31}$ Die Skandale mit bleihaltigem Spielzeug 2007 und

29 Vgl. www.misereor.de/fileadmin/user_upload/pflege_allgemein/pflege_startseite/2008_02_08_ pressemitteilung.pdf (gel. am 31.01.2009).

30 Vgl. „ICTI-Liste“ http://www.toy.de/news/index1761.html (gel. am 15.01.2009).

Zum Teil positionieren sich NGOs hier nicht eindeutig, etwa wenn behauptet wird, Lieferanten würden durch ihre Abnehmer bei zu wenig Unterstützung über Gebühr unter Druck gesetzt, Standards einzuhalten, wodurch wiederum das Betrugswesen bei den Produzenten gefördert werde (Kleinert 2008: 202). Im Gegenteil kann der Druck gar nicht groß genug sein, die ICTIStandards einzuhalten, da Auftragsentzug (also believable threats) durch die Markenfirmen letztendlich der größte Anreiz für Produzenten darstellt, Standards einzuhalten. 
2008 legen ein Zeugnis auch über die Skrupellosigkeit mancher chinesischer Unternehmer ab, für die nicht allein westliche Firmen verantwortlich gemacht werden können.

Ein Großteil der Mängel bei Sozialstandards wird letztlich jedoch immer wieder auf die sehr geringe Beteiligung der Arbeiter und entsprechenden Arbeitervertretungen in den Firmen zurückgeführt. Während ICTI bereits für mehr als 2500 Fabrikmanager Trainingsseminare durchgeführt hat, bleibt eine angemessene Aufklärung und Partizipation der Arbeiter die große Aufgabe für die Zukunft. So wurden bis Ende 2007 37000 Informationskarten in 25 Fabriken auf Probe ausgelegt, um Arbeiter über ihre Rechte zu informieren, außerdem würden bereits viele Arbeiter den telefonischen Service von ICTI nutzen, um sich zu informieren (vgl. ICTI 2008: 9). Dies kann sicherlich erst ein Anfang sein.

Insgesamt kann der ICTI-Kodex auf eine große Akzeptanz in der Industrie, im Handel und auch bei NGOs bauen, wenn bisherige Mängel abgestellt werden. Als „realistisches Ziel“" gibt ICTI eine Konvergenz von 95\% Abdeckung des Branchenkodexes bei allen Fabriken vor, während lediglich 5\% an spezifischen Punkten zusätzlich kontrolliert werden müssten (vgl. ICTI 2008: 9). Die Marktführer mussten erfahren, dass sie sich in einer interdependenten Situation mit vielen anderen Unternehmen befinden und sie ihre Standards unilateral nicht durchsetzen konnten. Dadurch, dass der Branchenstandard sowohl in Europa als auch den USA aus der Taufe gehoben wurde, und im Weltverband ein gemeinsames Dach hat, gibt es in der Spielzeugindustrie die einmalige Situation, dass sich eine global anerkannte Branchenvereinbarung herausbilden konnte, die nun, auch unter den Erfahrungen der jüngsten Skandale bei Spielzeugsicherheit, ihre Tragfähigkeit unter Beweis stellen muss. Zwar ist der Branchenkodex noch immer am Anfang seiner Implementierung mit allen Schwächen, die NGOs und Medienberichte immer wieder feststellen, dennoch gibt es wenig, was dagegen spräche, dass sich in der Spielzeugindustrie der ICTI-Kodex als ein dauerhafter Mindeststandard für Arbeitsschutz in den Spielzeugfabriken durchsetzt.

\section{Fazit: Zwei-Stufen-Kooperation in der Spielzeugindustrie}

Als zentrales Problem einer Branchenvereinbarung wurde zu Beginn eine geringe allgemeine Durchsetzungsfähigkeit, das Kooperationsproblem sowie eine damit eng zusammenhängende, geringe Attraktivität von Branchenstandards für Marktführer angesehen. Auf Marktführer kann jedoch bei Branchenvereinbarungen und zunehmender Wettbewerbskonzentration, die in vielen Branchen beobachtet wird, nicht verzichtet werden, was sich gerade auch in der Spielzeugindustrie zeigt. Zwar gibt es in dieser Branche viele tausend Akteure, jedoch nur relativ wenige, wirklich einflussreiche Unternehmen. Das Zustandekommen von Branchenvereinbarungen ist somit voraussetzungsvoll: Nur wenn sich die relativ wenigen Branchenführer eines Marktes zu gemeinsamen Beschlüssen durchringen, die sie kontrollieren und gegebenenfalls sanktionieren und wenn sie für die Bereitstellung eines institutionellen Rahmens durch Institutionalisierung sorgen, dem sich alle Akteure unterordnen, einschließlich der Marktführer und der vielen kleinen Unternehmen, können stabile Branchenvereinbarungen entstehen. Branchenstandards müssen zudem ein in etwa ebenso hohes Niveau haben wie die Standards der Marktführer, weil Branchenstandards nur so auch für sie attraktiv werden. 
Marktführer können über ihre Einkaufsmacht Kontrolle über ihre Lieferanten ausüben, was auch schon früher festgestellt wurde (vgl. Gereffi 1999; Frenkel 2001; Fichter/Sydow 2002). Jedoch haben viele Autoren die Renitenz der Fabriken unterschätzt, indem sie allein auf die Macht der Global Player verwiesen haben. Im Bereich Spielzeugsicherheit hat sich gezeigt, dass auch die Einkaufsmacht der Konzerne nicht verhindern konnte, dass sich chinesische Fabrikmanager unter Umgehung klarer gesetzlicher Vorgaben auf den Absatzmärkten hinwegsetzen. Durch Interdependenz können auch die zum Teil widersprüchlichen Standards der mächtigsten Unternehmen einer Branche nicht vollends durchgesetzt werden, weshalb Kooperationsanreize durch Koordinierung entstehen, wie das Beispiel Mattel zeigt. Koordinierung ist allerdings nur eine Vorraussetzung zur Durchsetzung von Branchenstandards. Hinzu kommt die Aufgabe, einen funktionierenden, institutionellen Rahmen zu schaffen, der die gemeinsamen Beschlüsse einhegt und dauerhafte Kooperation ermöglicht. Die Branchenvereinbarung wurde erst seit 2004 institutionell unterfüttert: Im Gegensatz zu eher machtbasierten Ansätzen, die Machtfaktoren und äußere Rahmenbedingungen zur Kooperationsinitiierung stärker berücksichtigen, hat ein institutionalistischer Ansatz (oder ein machtbasierter Ansatz, der, wie hier, durch einen institutionalistischen ergänzt wird) einen stärkeren Fokus auf die inhaltlichen Aspekte und die Qualität der Kooperation, die bei früheren Analysen von Gruppenstandards oder Branchenvereinbarungen zu wenig beachtet wurden.

Schematisch können die Prozesse, die in der Spielzeugindustrie ausgelöst wurden, als ein Zwei-Stufen-Modell abgebildet werden. Auf der ersten Stufe koordinieren die Marktführer und Einzelhandelsunternehmen ihre Standards. Hier ist keine umfassende Kooperation mehr notwendig, weil sich die Verhaltenskodizes in den letzten Jahren stark angenähert haben und sich nur noch im Detail unterscheiden (vgl. Biedermann 2007: 153 und 167). Der Konvergenzprozess der Industrie, hier spieltheoretisch als Koordinierung bezeichnet, ist weit vorangeschritten mit der klaren Zielvorgabe, global gültige Standards durchzusetzen. Die Koordination schafft die Voraussetzung einer umfassenden Institutionalisierung und Etablierung eines Kooperationsarrangements mit den vielen hundert kleineren Markenunternehmen und tausenden Produzenten. Die Institutionalisierung und Implementierung ist noch immer im Verbesserungsprozess, der von NGOs weiterhin kritisch und hilfreich begleitet wird. In der Spielzeugindustrie schufen die Marktführer durch Koordinierung ihrer Standards auf der ersten Stufe die Voraussetzung für den Durchbruch der Branchenvereinbarung auf der zweiten Stufe durch eine hegemonial unterstützte Institutionalisierung mittels des Weltverbands der Spielzeugindustrie. Gerade auch in der aktuellen Frage, wie kleinere und mittlere Unternehmen sozial verantwortlich in Schwellen- und Entwicklungsländern agieren können, sollten Branchenstandards größere Beachtung zuteil werden.

\section{Literaturverzeichnis}

Axelrod, R./ Keohane, R. O. (1985): Achieving Cooperation under Anarchy, Strategies and Institutions, in: World Politics, 38(1), 226-254.

Axelrod, R. (1995): Die Evolution der Kooperation, 3. Auflage, Oldenburg u. a.: Scientia Nova. 
Asia Monitor Resource Center (1998): Die Arbeitsbedingungen in der Spielwarenindustrie in China. Ein vorläufiger Untersuchungsbericht, Hongkong, http://eu-china.net/web/cms/ upload/pdf/materialien/wong_1998_arbeitsbedingungen_spielzeugindustrie_china.pdf.

Biedermann, R. (2006): From a Weak Letter of Intent to Prevalence - The Toy Industries Code of Conduct, in: Journal of Public Affairs, Special Issue on Corporate Social Responsibility, 6(3-4), 197-209.

Biedermann, R. (2007): Sozialstandards durch Private Governance. Zwei-Stufen-Kooperation in der globalen Spielzeugbranche, Baden-Baden: Nomos.

Brams, S. J. (1993): Theory of Moves, Cambridge Cambridge: University Press.

Brübl, T./ Debiebl, T./ Hamm, B./ Hummel, H./ Martens, J. (Hrsg.) (2001): Die Privatisierung der Weltpolitik. Entstaatlichung und Kommerzialisierung im Globalisierungsprozess, Bonn: Dietz.

Chan, A. (2003): A 'Race to the Bottom': Globalisation and China's Labour Standards, in: China Perspectives, 46(3-4), 41-49.

Cutler, A. C./ Haufler, V./ Porter, T. (Eds.) (1999): Private Authority and International Affairs, Albany: State University of New York Press.

Downs, G. W./ Rocke, D. M./ Barsoom, P. N. (1996): Is the Good News About Compliance Good News About Cooperation?, in: International Organization, 50(3), 73-102.

Egels-Zandén, N. (2007): Suppliers' Compliance with MNC' Codes of Conduct: Behind the Scenes at Chinese Toy Suppliers, in: Journal of Business Ethics, 75(1), 45-62.

Fichter, M./ Sydow, J. (2002): Using Networks Towards Global Labor Standards? Organizing Social Responsibility in Global Production Chains, in: Industrielle Beziehungen, 9(4), 357-380.

Frenkel, S. J. (2001): Globalization, Athletic Footwear Commodity Chains and Employment Relations in Southern China, in: Organization Studies, 22(4), 531-562.

Fuchs, D. (2004): The Role of Business in Global Governance, in: Schirm, A. (Ed.): New Rules for Global Markets. Public and Private Governance in the World Economy, Basingstoke: Palgrave Macmillan, 133-154.

Gereffi, G. (1999): International Trade and Industrial Upgrading in the Apparel Commodity Chain, in: Journal of International Economics, 48(1), 37-70.

Guinaudeau, P. (2008): Toy Markets in the World. 2008 Edition, ICTI.

Hall, R. B./ Biersteker, T. J. (Eds.) (2002): The Emergence of Private Authority in Global Governance, Cambridge: Cambridge University Press.

Hardin, R. (1982): Collective Action, Baltimore, MD: John Hopkins University Press.

Haufler, V. (2001): A Public Role for the Private Sector: Industry Self-Regulation in a Global Economy, Washington D. C.: Carnegie Endowment for International Peace.

Heidel, K/ Pater, S./ Piepel, K. (2002): Spielverderber. Das Geschäft mit dem Kinderspielzeug. Begleitb. z. Aktion ,Fair spielt', Aachen: Retap.

Holizinger, K./ Knill, C./ Sommerer, T. (2008): Environmental Policy Convergence: The Impact of International Harmonization, Transnational Communication, and Regulatory Competition, in: International Organization, 62(Herbst), 553-587.

Hong Kong Christian Industrial Committee (2000): BOM! Beware of Mickey. Disney Sweatshops in South China, Hongkong, http://www.cic.org.hk/download/whole\%20report2.doc.

Hong Kong Christian Industrial Committee (2001): How Hasbro, McDonald's, Mattel and Disney Manufacture their Toys. Report on the Labor Rights and Occupational Safety and 
Health Conditions of Toy Workers in Foreign Investment Enterprises in Southern Mainland China, Hongkong.

International Council of Toy Industries (2004): International Council of Toy Industries' Code of Business Practices. The CARE Process, Hong Kong Toys Council under the Auspices of the Federation of Hong Kong Industries, Hong Kong.

ICTI Care Foundation (2008): Creating Sustainable Change in the Workplace. Review of ICTI Care Process Activities through 2007, New York u. a.

Kindleberger, C. (1973): The World in Depression 1929-1939. Berkeley, Los Angeles: University of California Press.

Kleinert, U. (2006): Sozialstandards in der Spielzeugproduktion - Zwischenbilanz nach drei Jahren ICTI CARE-Prozess, in: Fonari, A. et al. (Hrsg.): Runder Tisch Bayern. Sozialund Umweltstandards bei Unternehmen, Augsburg und München: Eine-Welt-Netzwerk Bayern e.V. und Institut für Gesellschaftspolitik an der Hochschule für Philosophie, 143-150.

Kleinert, U. (2008): Sozialstandards in der Spielzeugproduktion: Höchste Zeit für mehr Verbindlichkeit, Eine Welt Netz Bayern, http://www.eineweltnetzbayern.de/globalisierung/ unternehmen/publikationen/csr2-spielzeugproduktion.pdf.

Krueger, D. A. (2008): The Ethics of Global Supply Chains in China - Convergences of East and West, in: Journal of Business Ethics, 79(1-2), 113-120.

Nölke, A. (2004): Transnational Private Authority and Corporate Governance, in: Schirm, A. (Ed.): New Rules for Global Markets. Public and Private Governance in the World Economy, Basingstoke: Palgrave Macmillan, 212-242.

Olson, M. (1992): Die Logik des kollektiven Handelns. Kollektivgüter und die Theorie der Gruppen, dritte Auflage, Tübingen: Mohr-Siebeck.

Piepel, K. (2002): Hinter verschlossenen Türen - Verhaltenskodizes in der Spielzeugindustrie, in: Scherer, A. G./ Blickle, K.-H./ Dietzfelbinger, D./ Hutter, G. (Hrsg.): Globalisierung und Sozialstandards, München/Mering: Hampp, 249-263.

Risse, T. (2005): Governance in Räumen begrenzter Staatlichkeit. „Failed States“ werden zum zentralen Problem der Weltpolitik, in: Internationale Politik, 60(9), 6-12.

Rivoli, P. (2003): Labor Standards in the Global Economy: Issues for Investors, in: Journal of Business Ethics, 43(3), 223-232.

Rowe, J. K. (2005): Corporate Social Responsibility as a Business Strategy, in: Lipschutz, R. D./ Rowe, J. K. (Eds.): Globalization, Governmentality and Global Politics. Regulation for the Rest of US?, London: Routledge, 121-160.

Rudder, E. C. (2008): Private Governance as Public Policy: A Paradigmatic Shift, in: The Journal of Politics, 70(4), 899-913.

Scherrer, C./ Greven, T. (2001): Global Rule for Trade. Codes of Conduct, Social Labeling, Workers' Rights Clauses, Münster: Westfälisches Dampfboot.

Sethi, P. (2003): Setting Global Standards, Guidelines for Creating Codes of Conduct in Multinational Corporations, New Jersey: John Wiley \& Sons.

Spar, D. L. (1994): The Cooperative Edge. The Internal Politics of International Cartels, Ithaca und London: Cornell University Press.

Stein, A. (1982): Coordination and Collaboration: Regimes in an Anarchic World, in: International Organization, 36(2), 299-324.

Tiemann, R. (1999): Ethische Branchenstandards. Ein Lösungsweg für Unternehmen aus moralischen Dilemmata, München/Mering: Hampp.

Wilkinson, R. (Ed.) (2005): The Global Governance Reader, London/New York: Routledge. 